\title{
Analog Performance Prediction Based on Archimedean Copulas Generation Algorithm
}

\author{
Kamel Beznia, Ahcène Bounceur, Reinhardt Euler \\ Lab-STICC Laboratory - European University of Britanny - University of Brest \\ 20, Avenue Victor Le Gorgeu \\ 29238, Brest, France \\ Email: \{Kamel.Beznia, Ahcene.Bounceur, Reinhardt.Euler $@ @$ univ-brest.fr
}

\begin{abstract}
Testing analog circuits is a complex and very time consuming task. In contrary to digital circuits, testing analog circuits needs different configurations, each of them targets a certain set of output parameters which are the performances and the test measures. One of the solutions to simplify the test task and optimize test time is the reduction of the number of tobe-tested performances by eliminating redundant ones. However, the main problem with such a solution is the identification of redundant performances. Traditional methods based on calculation of the correlation between different performances or on the defect level are shown to be not sufficient. This paper presents a new method based on the Archimedean copula generation algorithm. It predicts the performance value from each output parameter value based on the dependence (copula) between the two values. Therefore, different performances can be represented by a single output parameter; as a result, less test configurations are required. To validate the proposed approach, a CMOS imager with two performances and one test measure is used. The simulation results show that the two performances can be replaced by a single test measure. Industrial results are also reported to prove the superiority of the proposed approach. ${ }^{1}$
\end{abstract}

\section{INTRODUCTION}

In the functional test of analog circuits, the performances of each circuit are measured and verified to check if they are inside the specifications or not. If all the specifications are verified then the circuit is considered as functional (good) otherwise, it is considered as faulty, and thus it is rejected. However, if the number of the specifications is important, test time becomes more important and has a direct consequence on the test cost. For some analog circuit with a very high number of performances, the functional test is practically impossible to perform. Reducing this number is mandatory. The major problem in reducing the set of the performances is that it generates an important number of faulty circuits which will be sold and hereby increase the value of the defect level (proportion of faulty circuits that pass the test). The test engineers have to be careful in choosing a subset for which the defect level remains constant or increase only slightly. Until now, there is no efficient method that can reduce the set of the performances significantly. In this work we will try to eliminate a performance by predicting its values by observing another output parameter (test measure and performance). The idea is based on using the dependency structure (copula) of

\footnotetext{
${ }^{1}$ This work is supported by the ISSTB (Institut Supérieur des Sciences et Technologies de Brest), France.
}

the output parameters. A copula is a multivariate uniform distribution. For the case of a two dimensional copula, if a set of values of the first dimension is generated from the univariate uniform distribution than the values of the second dimension can be obtained by applying a simple transformation function. One of the advantages of this method is that it can consider a non linear dependency between the output parameters. Let us consider a performance that we want to predict from an output parameter for an initial set of produced circuits. Some of these circuits will be taken in order to calculate the copula between the considered output parameter and this performance. Then, this copula will be used to predict the performance of the other circuits of the initial set by calculating only the value of the output parameter. This approach will be detailed in this paper. In the next section, we will review some previous work. In section III, we will introduce the Archimedean copulas theory. In Section IV, we will present the proposed methodology used to predict any performance from any output parameter. Section $\mathrm{V}$ will present the application of this method for the case study of a CMOS imager. Finally, we conclude the paper with some future work.

\section{Previous Work}

Several methods are proposed to reduce the time required in the functional test. Some methods are based on ordering the performances [1] [2] [3]. In these methods the first tested performances are those that have a high probability to detect faulty circuits. Decision binary trees have been used in [4], where binary classifiers are used to compact the complete test set by eliminating redundant tests. In [5] a subset of specifications is chosen based on the defect level test metric. Other methods are based on ATPG (Automatic Test Pattern Generation) algorithms used to predict the performances from the circuit responses [6]. In [7] a regression model calculated from a subset of tests is used to predict other tests outside of the considered subset. With the same idea of using regression models, [8] proposed a technique that predicts the values of the performances from a small set of test measures. The method proposed in [9] tries to predict performances based on models built on the correlation of the output parameters. In [10] a fast exploration of the functional test space is used to determine redundancy in functional tests. Machine learning has also been 
used recently in [11] to learn the intricate mappings between a subset of functional tests and direct pass/fail decisions.

The idea that the non linear dependency between the output parameters exist and can be of major importance in circuit behavior leads us to consider the copulas theory to track these dependencies. In [12] we used the copulas theory to generate a large sample of circuits from a small one, in order to estimate the test metrics and to fix optimally the test limits with high precision. In this work, we propose a new method where any performance can be predicted from any output parameter by estimating their copula. This copula can be estimated from an initial small subset of produced circuits. Then, a new copula with the same parameters will be generated using the values of the considered output parameter. This new copula will then be used to predict the performance values of all the circuits. The problem of fixing the test limits is not reported here because the limits of the predicted performance are the same as the one of the initial performance (i.e., the specification). Also, the test limits can only be fixed at the design stage from the Monte Carlo simulation. However, the proposed method can be applied directly to a real set of produced circuits.

\section{ARChIMEDEAN COPUlas TheORY}

\section{A. Estimating the copula}

The theory of the Copulas will not be described formally in this paper. Basic definitions and properties of copula functions are presented in [13]. A succinct introduction to copulas for test metrics estimation is given in [12][14]. To illustrate how to calculate copulas from any sample, we propose to consider the bivariate example of Figure 1.

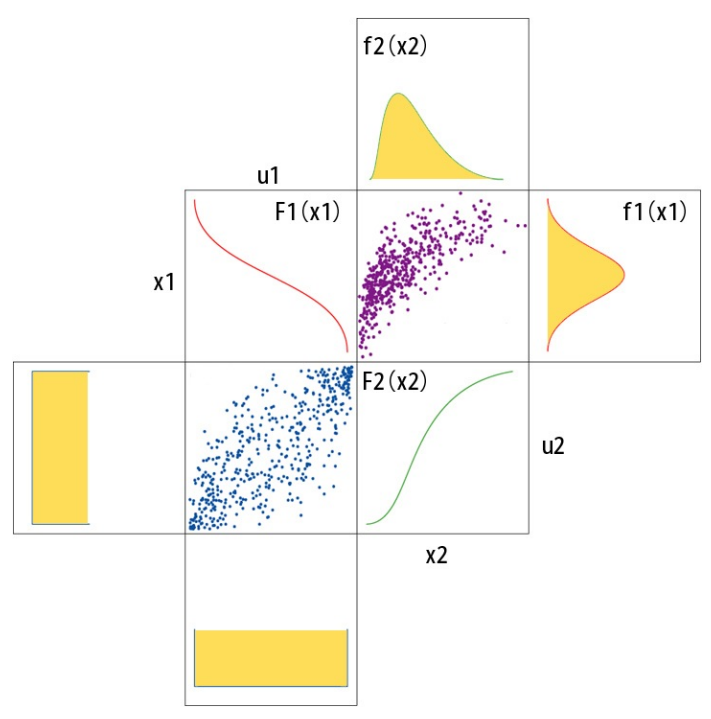

Fig. 1. Calculating the copula from a population.

The scatter plot of a bivariate random vector $X=\left(X_{1}, X_{2}\right)$ is shown in the upper right corner of Figure 1. To separate the dependencies between these two random variables from their marginal distributions, we apply the transformation $u_{i}=$ $F_{i}\left(x_{i}\right)$, where each initial sample point $\left(x_{1}, x_{2}\right)$ is transformed into a new point $\left(u_{1}, u_{2}\right)$ using the marginal CDF (Cumulative Density Function) of each variable ( $F_{1}$ for $x_{1}$ and $F_{2}$ for $x_{2}$ ). The result of this transformation is the bivariate random vector $U=\left(U_{1}, U_{2}\right)$ shown in the lower left corner. This new sample distribution corresponds to an empirical copula for which the marginal distributions are uniform. This complete and scalefree description of dependence is more suitable to be fit to well known multivariate parametric laws called copula.

\section{B. Archimedean copulas}

Archimedean copulas include a large variety of copula families that can be easily constructed to model non linear dependencies and non elliptical distributions. For example, Archimedean copulas can describe asymmetric dependencies, where the dependence coefficients in the upper and the lower tails are different. Some basics about Archimedean copulas will be discussed in this section. The dependence coefficient Kendall's $\tau$ will be considered instead of the classical linear correlation factor $\rho$. An estimator $\hat{\tau}$ of this coefficient is calculated as follows:

$$
\hat{\tau}=\frac{2}{n(n-1)} \sum_{i<j} \operatorname{sgn}\left[\left(x_{i}-x_{j}\right)\left(y_{i}-y_{j}\right)\right], \quad i, j=1, \ldots, n
$$

where

$$
\operatorname{sgn}(z)=\left\{\begin{array}{cc}
1 & \text { if } z \geq 0 \\
-1 & \text { if } z<0
\end{array}\right.
$$

and, $\left(x_{1}, y_{1}\right), \ldots,\left(x_{n}, y_{n}\right)$ are $n$ observations from a vector $(X, Y)$ of continuous random variables.

A two dimensional Archimedean copula is a function $C$ from $[0,1]^{2}$ to $[0,1]$ which has the following form:

$C\left(u_{1}, u_{2}\right)=\left\{\begin{array}{cc}\varphi^{-1}\left(\varphi\left(u_{1}\right)+\varphi\left(u_{2}\right)\right) & \text { if } \\ 0 & \varphi\left(u_{1}\right)+\varphi\left(u_{2}\right) \leq \varphi(0) \\ \text { otherwise }\end{array}\right.$

where $\varphi$ is called the generator function of the Archimedean copula. Notice that the generator function allows to write the copula as a sum of functions of the marginal distributions. For $0 \leq u \leq 1, \varphi$ is defined as $\varphi(1)=0, \varphi^{\prime}(u)<0$ and, $\varphi^{\prime \prime}(u)>0$.

In the following, we will present two families of Archimedean copulas. The first one is the Gumbel copula family and the second one is the Clayton copula family. These copulas are used in the test vehicle in order to predict performance values from those of the output parameters.

\section{1) Gumbel Copula:}

The Gumbel copula [13] is an Archimedean copula whose generator function is defined as:

$$
\varphi(u)=(-\ln (u))^{\theta}
$$

with, $\theta \in[1,+\infty[$. For the two dimensional case its associated function $C\left(u_{1}, u_{2}\right)$ can be written as follows:

$$
C\left(u_{1}, u_{2}\right)=\exp \left(-\left[\left(-\ln \left(u_{1}\right)\right)^{\theta}+\left(-\ln \left(u_{2}\right)\right)^{\theta}\right]^{1 / \theta}\right)
$$


The parameter $\theta$ depends on Kendall's $\tau$ and is calculated as follows:

$$
\theta=\frac{1}{1-\tau}
$$

Figure 3(a) shows the CDF of the Gumbel copula with a parameter $\theta=1.26$ and Figure 2(b) shows a set of 1000 samples generated from this copula.

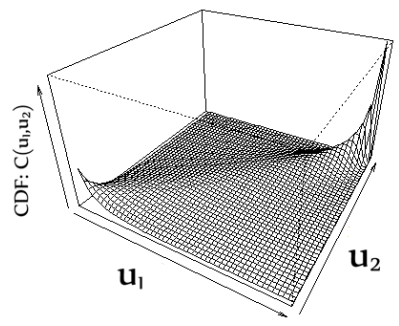

(a)

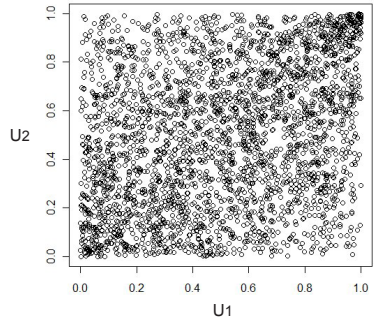

(b)
Fig. 2. (a) $\mathrm{CDF}$ of a Gumbel copula with $\theta=1.26$, (b) 1000 samples generated from the Gumbel copula.

\section{2) Clayton Copula:}

The Clayton copula [16] [17] is an Archimedean copula whose generator function is defined as:

$$
\varphi(u)=\frac{1}{\theta}\left(u^{-\theta}-1\right)
$$

with, $\theta \in]-1,0[\cup] 0, \infty[$. For the two dimensional case its associated function $C\left(u_{1}, u_{2}\right)$ can be written as follows:

$$
C\left(u_{1}, u_{2}\right)=\max \left(\left[u_{1}^{-\theta}+u_{2}^{-\theta}-1\right]^{-1 / \theta}, 0\right)
$$

The generalized form of this equation is given in [13]. The parameter $\theta$ depends on Kendall's $\tau$ and is calculated as follows:

$$
\theta=-\frac{2 \tau}{\tau-1}
$$

Figure 3(a) shows the CDF of the Clayton copula with a parameter $\theta=-0.63$ and Figure 3(b) shows a set of 1000 samples generated from this copula.

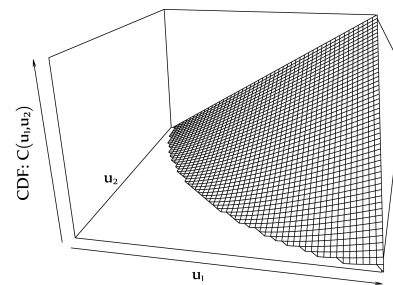

(a)

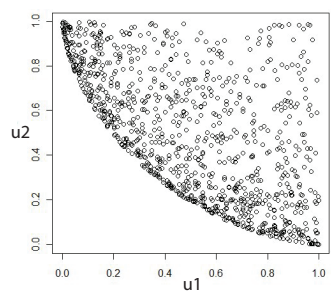

(b)

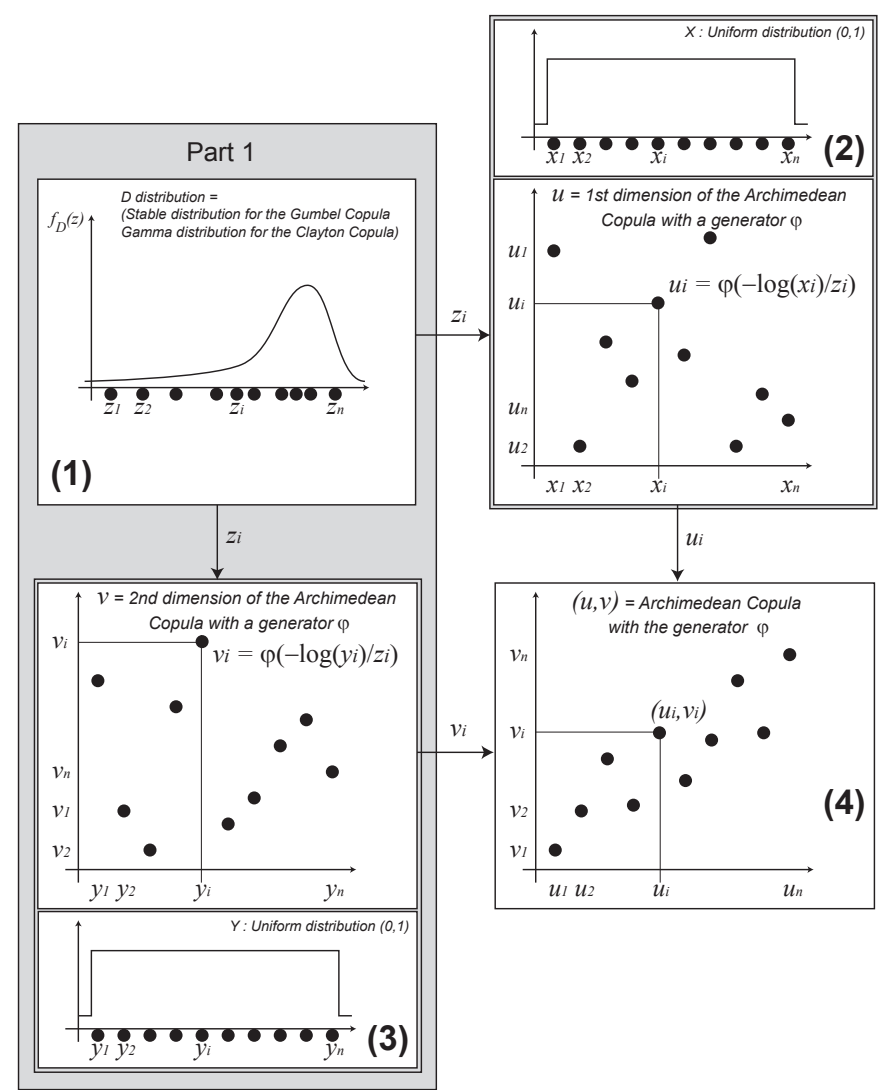

3) Archimedean copulas generation algorithm: To generate $n$ samples from a 2-dimensional Archimedean copula with the generator $\varphi$ we use the following Marshall \& Olkin algorithm [15] which is illustrated by Figure 4:

(1) Generate $n$ independent variables $z_{1}, \ldots, z_{n}$ with distribution function $D$ ( $Z \sim$ Gamma distribution for the Clayton copula and $Z \sim$ Positive Stable distribution for the Gumbel copula, cf. Table I).

(2) Generate $n$ independent variables $x_{1}, \ldots, x_{n}$ with the uniform distribution $X \sim \mathcal{U}[0,1]$.

(3) Generate $n$ independent variables $y_{1}, \ldots, y_{n}$ with the uniform distribution $Y \sim \mathcal{U}[0,1]$.

(4) Return $\left(\left(u_{1}, v_{1}\right), \ldots,\left(u_{n}, v_{n}\right)\right)$, where, $u_{i}=\varphi\left(-\ln \left(x_{i}\right) / z_{i}\right)$ and $v_{i}=\varphi\left(-\ln \left(y_{i}\right) / z_{i}\right)$, $i \in 1, \ldots, n$.

The parameters of the distribution $D$ used in this algorithm are given in the following Table I.

\begin{tabular}{|c|c|c|}
\hline Copula family & Distribution D & Parameters of D \\
\hline Clayton & Gamma $(\Gamma)$ & $\Gamma(1 / \theta, 1)$ \\
Gumbel & Positive Stable (St) & $S t\left(1 / \theta, 1,(\cos (\pi /(2 \theta)))^{\theta}, 0\right)$ \\
\hline
\end{tabular}

\section{TABLE I}

PARAMETERS OF THE $D$ Distribution.

Fig. 3. (a) $\mathrm{CDF}$ of a Clayton copula with $\theta=-0.63$, (b) 1000 samples generated from the Clayton copula.
Fig. 4. Illustration of the Marshall \& Olkin algorithm. 


\section{PERFormance PREDICTION METHOD}

The algorithm presented above is the main part of the proposed method used to predict performance values from values of any output parameter. Figure 5 shows the steps of the proposed method. Let $S$ be a set of $n$ produced circuits, $P 1$ a considered performance and $T$ the output parameter which will be used to predict the values of $P 1$. We will consider a subset of only $m \ll n$ circuits. From this subset, we estimate the copula $C 1$ of $P 1$ and $T$, i.e., $C 1=(V, W 1)$, where $V=C D F(T)$ and $W 1=C D F(P 1)$. Note that, $V$ and $W 1$ have a uniform distribution $\mathcal{U}[0,1]$. The algorithm of Marshall \& Olkin presented above can be used to generate another copula $C 2(V, W 2)$ with the same parameters as those of $C 1(V, W 1)$. We can consider this procedure as a method that predicts the values of $W 1$ knowing the values of $V$. Therefore, using $C 2$ we can then predict the values of $W 1$ of all the circuits. Finally, we can calculate the values of the performance $P 1$ of all the circuits by calculating the inverse CDF of $W 2$, i.e., $P 1 \approx P 2=C D F^{-1}(W 2)$.

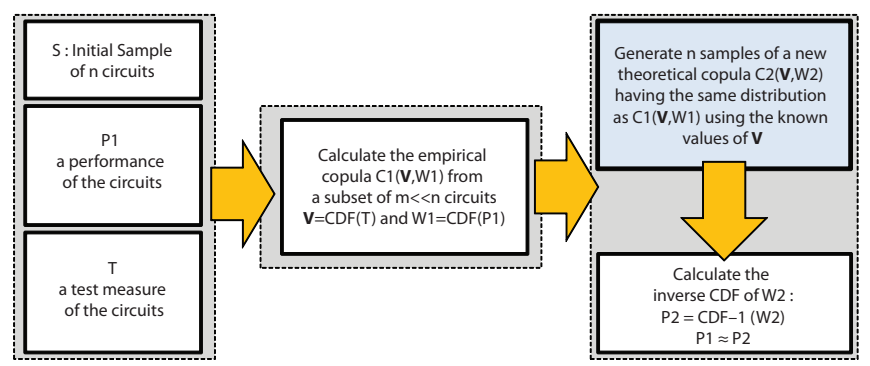

Fig. 5. Performance prediction method.

\section{TEST VEHICLE}

The considered case-study is a BIST technique for a CMOS imager presented in [18]. The analog and mixed-signal parts of the imager include a large pixel matrix, the column amplifiers and the data converters. The pixel matrix usually composed of millions of pixels is typically read line by line through the column amplifiers. Figure 6 shows the pixel structure composed by PMOS transistors and a photodiode. This type of pixel gives a logarithmic relationship between the output voltage $\left(V_{p h}\right)$ and the incoming light (represented as the photogenerated current $I_{p h}$ ).

The main performance measured for the pixel matrix and the column amplifier are the Fixed Pattern Noise (FPN). The FPN represents the difference that exists between two pixels (or two column amplifiers) under constant illumination. Different light sources are used to measure this noise. Two major kinds of FPN are measured: Pixel Response Non Uniformity (PRNU) that is obtained by using light sources and Dark Signal Non Uniformity (DSNU) that is obtained under dark conditions.

The BIST technique consists of the application of a voltage pulse at the anode of the photodiode, to measure the output voltage VA of the pixel. The whole analog ground of the pixel is externally pulsed (not shown in Figure 6). This electrical measurement is performed very fast, and the output

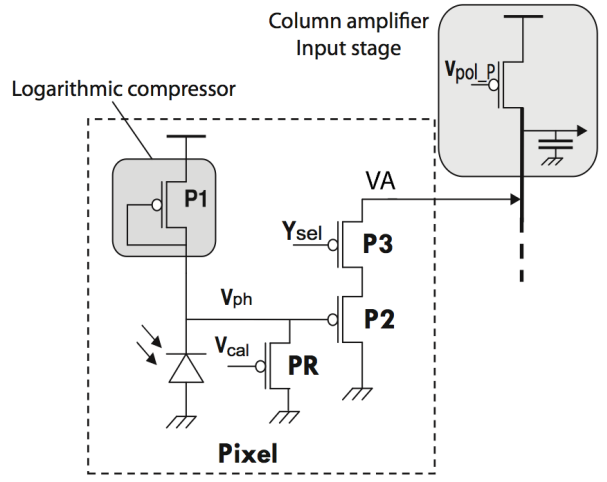

Fig. 6. Logarithmic pixel structure.

measurement is thus not dependent on the incoming light. This BIST measurement is intended to capture the major sources of DSNU, such as transistor mismatches.

The specifications of the two performances are $P R N U \in$ $[-0.0306,0.0306]$ Volt, and $D S N U \in[-0.0216,0.0216]$ Volt are fixed at $2 \sigma$ which leads to a yield of $\simeq 460 \mathrm{dppm}$ (defect part per million, i.e. 460 pixels out of specifications in 1 million). Since we have only 16383 pixels, if the specifications are fixed to $3 \sigma$ or more, only a few faulty circuits $(\approx 0)$ will be generated. That is why we choose to fix the specifications at $2 \sigma$ to generate an important number of defect circuits. Then, the proposed method can be evaluated efficiently by comparing the original yield calculated from the initial performances and the predicted yield obtained from the predicted performances.

\section{A. Performance prediction of the CMOS imager}

In our case study, we will try to predict the values of the PRNU and the DSNU of each circuit just by calculating the values of VA. Thus, we will take a subset of $m=3000$ circuits from which the empirical copula of VA and PRNU (or DSNU) will be estimated. This one will then be used to generate another theoretical copula with the same parameters by taking into account the values of VA. Finally, this new copula will be used to predict the performance values from the values of the VA. For more clarity, we detail this method in the following steps :

1) Let $S$ be the set of 16383 pixels

2) Let $T=V A$ be the considered test measure

3) Let $P 1=P R N U$ (or $P 1=D S N U$ ) be the performance that we want to predict from VA

4) Consider a small subset $S^{\prime} \subset S$ of 3000 circuits

5) Estimate the empirical copula $C 1(V, W 1)$ in this subset $\left(S^{\prime}\right)$, where $V$ is the CDF of $T(V=C D F(T))$ and $W 1$ is the CDF of $P 1(W 1=C D F(P 1))$.

6) Generate a set of 16383 values from a new theoretical copula $C 2(V, W 2)$ with the same parameters as $C 1$ using the Marshall \& Olkin algorithm presented in Section III-B.3. Note that the first dimension of this copula is the 16383 values of $V$, i.e., the CDF of $T$ calculated from the set $S$. 
7) Calculate $P 2=C D F^{-1}(W 2)$. The values of $P 2$ are close to the values of $P 1$ predicted from $V$.

\section{B. Results}

We will use the steps presented above to predict the values of the first performance PRNU from those of the test measure VA. The same steps will be used to predict the values of the second performance DSNU from the test measure VA.

First, we will estimate the empirical copula of PRNU and VA by calculating $W 1$, the CDF of PRNU and $V$, the CDF of VA from a subset of 3000 pixels. Figure 7 shows the obtained copula. If we compare this copula to the one of Figure 2(b) we conclude that it is a Gumbel copula. This comparison has been validated using a goodness of fit test of copulas, not discussed here for sake of simplicity. This copula is characterized by the parameter $\theta$ which is calculated from Kendall's $\tau$ correlation factor using the Equation (5). In our case, $\theta$ is equal to 1.26.

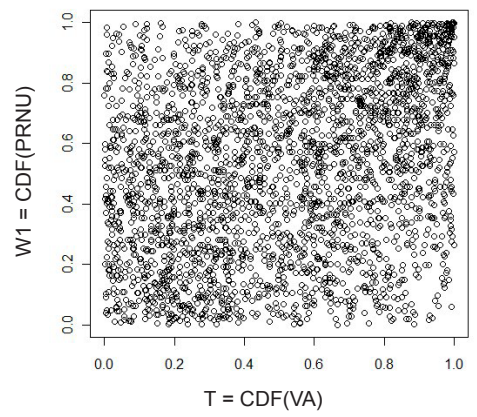

Fig. 7. 3000 samples of the empirical copula of the PRNU and VA.

Now, instead of considering only the 3000 circuits, we will take into consideration all the 16383 pixels. We will generate 16383 samples from the new theoretical Gumbel copula $C 2(V, W 2)$ with the parameter $\theta=1.26$ in which the first dimension is equal to the values of the CDF of VA. Figure 8 shows the obtained copula.

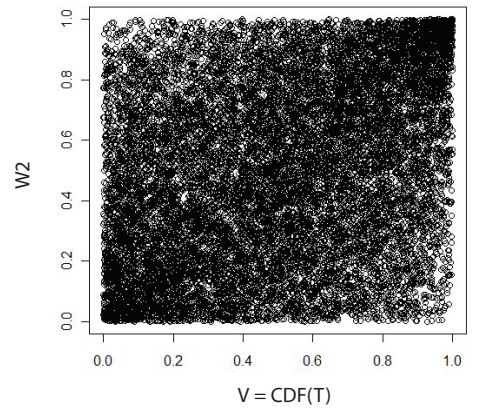

Fig. 8. 16000 samples generated from the theoretical Gumbel copula.

Then, by calculating the inverse CDF of $W 2$ we will obtain the values of $P 2$, the predicted performance, which is close to the PRNU. Figure 9 shows the joint distribution of the PRNU and the test measure VA obtained from the initial performance (in black o) and from the predicted performance (in gray + ). It is clear that the two distributions are very close.

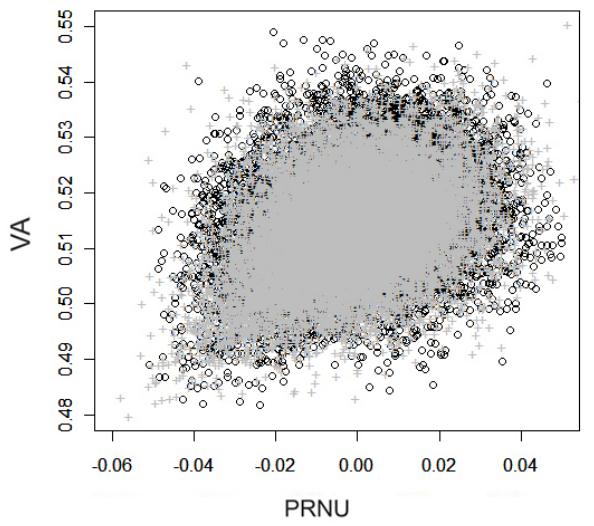

Fig. 9. The joint distribution of the test measure VA and the initial performance (black o) compared to the joint distribution of the test measure VA and the predicted performance (gray + ).

Figure 10 shows the first 200 values of the initial performance (in black*) and those of the predicted performances for the same circuits (in red + ). As we can see, the values are very close to each other. We have also compared the Yield (proportion of the good circuits) obtained in each case. It is equal to $Y 1=462 d p p m$ in the initial PRNU and $Y 2=474 d p p m$ in the predicted PRNU.

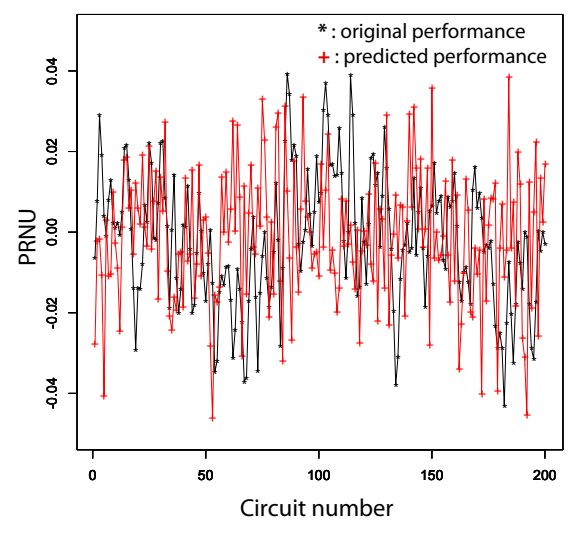

Fig. 10. A set of 200 values of the initial performances (black *) vs. those of the predicted performances (red + ).

For the case of the second performance DSNU, we used the same procedure as presented above. Figure 11 shows the obtained copula between the DSNU and the test measure VA. If we compare it to the one of Figure 3(b) we conclude that it is a Clayton copula. A goodness of fit test of copula is used to validate this conclusion.

Following the procedure presented above for the case of the Gumbel copula, Figure 12 shows the values of the initial DSNU and those of the predicted DSNU. In this figure, only the first 200 values are drawn. It is clear that the two graphs are close. The Yield estimated in each case is equal to $Y 1=$ $466 d p p m$ in the initial DSNU and $Y 2=454 d p p m$ in the 


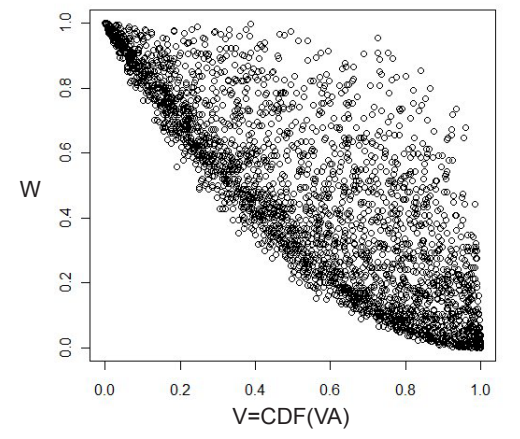

Fig. 11. 3000 samples of the empirical copula of the DSNU and VA.

predicted DSNU.

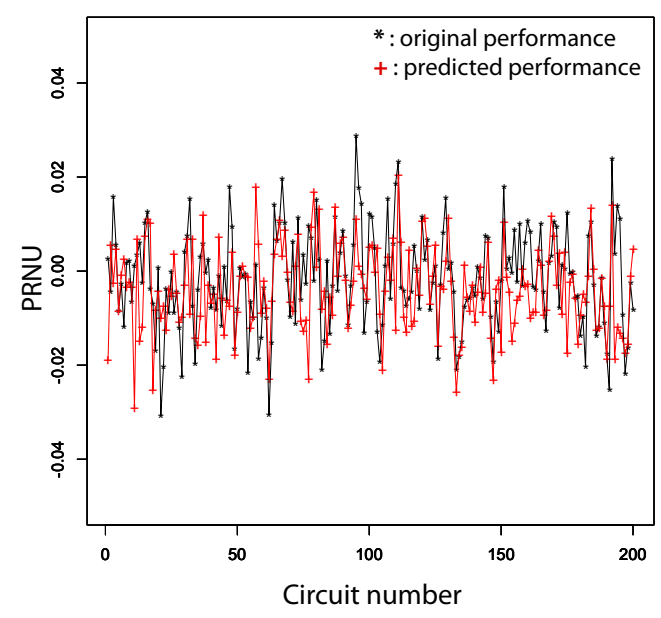

Fig. 12. A set of 200 values of the initial performances (black *) vs. those of the predicted performances (red + ).

\section{CONCLUSIONS}

This paper has presented a new method for the prediction of performance values from those of the output parameters (test measures and performances) in a set of produced circuits. The proposed method is based on the Archimedean copula generation algorithm. Marshall \& Olkin's algorithm is used to generate from a uniform distribution another uniform distribution to obtain a dependent couple that follows any targeted copula. This algorithm has also been used to generate from the $\mathrm{CDF}$ of any output parameter, which is uniform, the CDF of any performance by estimating their copula. The method has been applied to a set of pixels of a CMOS imager with two performances PRNU and DSNU and one test measure VA. The obtained results based on the estimation of the Yield shows that the predicted performance is very close to the original one. Future work will target the use of the same method to fix the test limits without calculating the test metrics.

\begin{tabular}{|l|l|l|l|}
\hline Copule & \multicolumn{2}{|c|}{ tau de dépendance } \\
\hline$\alpha_{1}$ & & & \\
\hline$\alpha_{1}$ & & & \\
\hline$\alpha_{1}$ & & & \\
\hline
\end{tabular}

\section{ACKNOWLEDGEMENTS}

The authors would like to acknowledge the cooperation and the help of Salvador Mir, TIMA Laboratory, Grenoble, France, and Bernard Pottier, Lab-STICC, Brest, France.

\section{REFERENCES}

[1] L. Milor and A. L. Sangiovanni-Vincentelli, Minimizing production test time to detect faults in analog circuits, IEEE Transactions ComputerAided Design of Integrated Circuits and Systems, vol. 13, no. 6, pp. 796.813, 1994.

[2] Nourredine Akkouche, Salvador Mir and Emmanuel Simeu, Ordering of Functional Tests Based on Parametric Defect Level Estimation, 28th IEEE VLSI Test Symposium (VTS'10), Santa Cruz, California, USA, April 2010.

[3] S. D. Huss and R. S. Gyurcsik. Optimal ordering of analog integrated circuit tests to minimize test time, 28th ACM/IEEE Design Automation Conference, 1991, pp. 494-499.

[4] S. Biswas and R. D. Blanton. Statistical test compaction using binary decision trees, IEEE Design and Test of Computers, vol. 23, no. 6, 2006, pp. 452-462.

[5] N. Akkouche, A. Bounceur, S. Mir, and E. Simeu, Minimization of functional tests by statistical modelling of analogue circuits, Proceedings of International Conference on Design and Technology of Integrated Systems in nanoscale era (DTIS), 2007, pp. 35-40.

[6] R. Voorakaranam and A. Chatterjee, Test Generation for Accurate Prediction of Analog Specifications, Proceeding of VTS, 2000, pp.137-142.

[7] J. B. Brockman and S.W. Director Predictive subset testing: optimizing IC parametric performance testing for quality, cost, and yield, IEEE Transactions on Semiconductor Manufacturing, vol. 2, no. 3, pp. 104113, 1989.

[8] S. Battacharya, A. Halder, G. Srinivasan, and A. Chatterjee. Alternate testing of RF transceivers using optimized test stimulus for accurate prediction of system specifications. In Journal of Electronic Testing : Theory and Applications (JETTA), vol. 21, 2005, 323-339.

[9] Jungran Lee, Walker D.M.H., Milor L., Yeng Peng, Hill G. IC performance prediction for test cost reduction, IEEE International Symposium on Semiconductor Manufacturing Conference Proceedings, Santa Clara, CA, USA, 1999, 111-114.

[10] Gomes A. V. and A. Chatterjee. Distance constrained dimensionality reduction for parametric fault test generator, Proceeding of Asia Test Symposium, 2001, pp. 411-416.

[11] H. G. Stratigopoulos, P. Drineas, M. Slamani, and Y. Makris, Non-RF to RF Test Correlation Using Learning Machines: A Case Study, 25th Proceedings IEEE VLSI Test Symposium, 2007, pp. 9-14.

[12] A. Bounceur, S. Mir and H-G. Stratigopoulos. Estimation of analog parametric test metrics using copulas. IEEE Transactions Computer-Aided Design of Integrated Circuits and Systems, September 2011, volume 30, number 09, ITCSDI, ISSN 0278-0070.

[13] R.-B. Nelsen. An Introduction to Copulas. Lecture Notes in Statistics. Springer, New York, 1999.

[14] K.Beznia A. Bounceur and S. Mir. R.Euler, Parametric test metrics estimation using non-Gaussian copulas, In IEEE 17th International MixedSignals, Sensors and Systems Test Workshop (IMS3TW), Santa Barbara, California, USA, 2011, May 16-18.

[15] A. W Marshall and I. Olkin. Families of multivariate distributions, Journal of the American Statistical Association, 1988, 83-403.

[16] Clayton, D. G. A model for association in bivariate life tables and its application in epidemiological studies of familial tendency in chronic disease incidence. Biometrika, 1978, 65: 141-151.

[17] Genest, C. and MacKay, R. J. (1986). Copules archimédiennes et familles de lois bidimensionnelles dont les marges sont données. Canadian Journal of Statisttics, 14: 145-159.

[18] Livier Lizarraga, Salvador Mir, Gilles Sicard, Experimental Validation of a BIST Technique for CMOS Active Pixel Sensors, In the 27th IEEE VLSI Test Symposium VTS, 2009, pp.189-194. 